\title{
DECENTRALIZED RECONSTRUCTION FROM COMPRESSIVE RANDOM PROJECTIONS DRIVEN BY PRINCIPAL COMPONENTS
}

\author{
James E. Fowler \\ Department of Electrical and Computer Engineering \\ Distributed Analytics and Security Institute \& Geosystems Research Institute \\ Mississippi State University, USA
}

\begin{abstract}
The decentralized reconstruction of data acquired in a sensor network via compressive random projections is considered. Assuming each node acquires a signal while simultaneously reducing its dimensionality, the proposed decentralized reconstruction recovers each signal to its original dimensionality with the reconstruction process being distributed across the network such that each node performs limited computation with limited communication with its neighboring nodes. In contrast to prior decentralized reconstructions driven by sparsity-based compressed-sensing techniques, the proposed approach employs reconstruction based on principal component analysis using an iterative consensus algorithm to calculate the required covariance across the network. Experimental results reveal that the performance of the proposed decentralized reconstruction approaches that of the original centralized algorithm as the number of consensus iterations increases.
\end{abstract}

Index Terms — random projections, principal component analysis, decentralized reconstruction, sensor networks

\section{INTRODUCTION}

The past decade has witnessed an explosion of interest in signal-acquisition paradigms in which a signal is sensed and reduced in dimensionality simultaneously by a sensing device. Much of this interest has arisen as the result of the advent of sparsity-driven compressed-sensing (CS) reconstructions, although other formulations are possible, such as the reconstructions of $[1,2]$ based on principal component analysis (PCA). Regardless of specifics of the recovery side of the system, the appeal of CS and similar reconstructions stems from the promise of lightweight sensing architectures which typically implement simultaneous sensing and dimensionality reduction via compressive random projections directly in the hardware of the sensing device. Such compressive random-projection sensing of signals is particularly well suited to sensing environments what are severely constrained in resources such as computation, storage, power consumption, and communication bandwidth. In essence, this sensing paradigm effects a shift in resource burden from the sensing side of the system to the reconstruction side in contrast to traditional communication systems which feature resource-intensive encoders.

It can be argued that a sensor network is a natural fit for simultaneous sensing and dimensionality reduction since the individual sensor nodes are often highly constrained in their access to computation, memory, power, and other resources. In such a sensor network, each sensor node individually acquires a signal directly in a reduced dimensionality, and the resulting signal measurements are then feed into a reconstruction process to recover the signal in its original dimensionality. The most straightforward paradigm for accomplishing this reconstruction is via the transmission of the signal measurements from all the individual sensor nodes to some central node, or "fusion center." In contrast to the resourceconstrained sensors, this fusion center is assumed to be a powerful, resource-rich processor capable of handling the intensive demands entailed by the reconstruction of the entire collection of sensed signals. Such a distributed sensing framework employing a CS-based reconstruction at the fusion center was proposed in [3-6], for example.

However, it has been recognized (e.g., [7,8]) that such centralized reconstruction is not always feasible. In certain settings, a fusion center may not be available, or may be insufficiently powerful to handle the reconstruction for a network of extremely large size; alternatively, the network may not have sufficient bandwidth to handle the communication of the signal measurements toward a single point, again, particularly if the network has an exceedingly large number of nodes. In such situations, a decentralized reconstruction in which the reconstruction computation is distributed throughout the entirety of the network, with each sensor node performing limited computation with limited communication with neighboring nodes, may be the preferred-or even the only possiblesolution. Such decentralized reconstruction has been proposed previously for CS as well (e.g., [7-12]).

In this paper, we also consider a decentralized reconstruction of signals acquired with reduced dimensionality in a sensor network. However, in contrast with prior work such as [3-12], we employ a PCA-based reconstruction rather than a 
reconstruction using sparsity-based $\mathrm{CS}$, as it has been demonstrated $[1,2]$ that such PCA-based reconstructions can significantly outperform their corresponding CS-based counterparts. Specifically, as the primary contribution of the present work, we formulate a decentralized version of the PCA-driven reconstruction proposed in [2]; at the core of the proposed process is a covariance estimate by iterative consensus. Experimental results reveal that the proposed decentralized variant approaches the performance of the original centralized algorithm as the number of consensus iterations increases.

Before detailing specifics, we note that our proposed approach bears some resemblance to several formulations described as "distributed compressed sensing" (DCS) in prior literature. Perhaps most commonly, DCS has referred to a framework originating with [3] in which sensors acquire multiple distinct signals that are jointly sparse, but with centralized reconstruction of the entire collection of signals taking place in a fusion center (see also, e.g., [4-6]). On the other hand, $[7,8]$ describe an alternate DCS framework in which sensors acquire multiple noise-corrupted versions of the same signal, but with decentralized reconstruction of the single signal being sensed. However, the approach we consider here is more in the spirit of DCS as formulated in $[11,12]$ which consider sensing of distinct yet mutually correlated signals as in [3] but use a decentralized reconstruction in the style of [7, 8]. However, in contrast to $[11,12]$, our approach eschews CS in favor of a PCA-based reconstruction that we describe next, first in its original centralized form and then in our proposed decentralized variant.

\section{BACKGROUND}

Consider a network of $M$ sensors, each acquiring a single $N$-dimensional signal $\mathbf{x}_{m} \in \Re^{N}$. However, further consider the situation in which the signal acquisition is "compressive" in nature; i.e., each sensor in the network simultaneously acquires its signal and reduces dimensionality via an $N \times K$ measurement matrix $\mathbf{P}_{m}$ such that

$$
\mathbf{y}_{m}=\mathbf{P}_{m}^{T} \mathbf{x}_{m}
$$

where $\mathbf{y}_{m} \in \Re^{K}$, and $K \ll N$. The subrate is defined as the ratio $K / N$. We assume that the original signal vectors $\mathbf{x}_{m}$ are mutually correlated. We assume also that $\mathbf{P}_{m}$ is different for each sensor and is known only to sensor $m$ and not to the other sensors in the network. Finally, each $\mathbf{P}_{m}$ is orthonormal in the sense that $\mathbf{P}_{m}^{T} \mathbf{P}_{m}=\mathbf{I}$. The task at hand then is to reconstruct signal $\mathbf{x}_{m}$ as approximation $\hat{\mathbf{x}}_{m}$ from measurement $\mathbf{y}_{m}$ at each sensor node.

The straightforward approach is to use a centralized reconstruction framework. That is, each sensor sends its measurement $\mathbf{y}_{m}$ (and $\mathbf{P}_{m}$ as well) to some central node, or fusion center, which then applies some suitable reconstruction algorithm on the entire dataset to produce the collection of $\hat{\mathbf{x}}_{m}$ vectors. A simplistic approach would be to apply any of a number of CS reconstructions individually to the vectors, although CS reconstructions designed specifically for collections of correlated vectors (e.g., [3, 13]) would likely perform significantly better. However, it has been demonstrated that reconstructions based on PCA $[1,2]$ can outperform CS-based reconstructions by a wide margin.

For example, the PCA-based reconstruction proposed in [2] approximates the covariance of the dataset as

$$
\hat{\boldsymbol{\Sigma}}=\frac{1}{M} \sum_{m=1}^{M} \mathbf{P}_{m} \mathbf{y}_{m} \mathbf{y}_{m}^{T} \mathbf{P}_{m}^{T}
$$

after which the first principal component, $\boldsymbol{\psi}_{1}$, of the dataset $\left\{\mathbf{x}_{m}\right\}_{m=1}^{M}$ is approximated by the first eigenvector, $\hat{\boldsymbol{\psi}}_{1}$, of $\hat{\boldsymbol{\Sigma}}$. Subsequent principal components are then determined via a deflation procedure which first produces a least-squares estimate [1] of the dataset,

$$
\hat{\mathbf{x}}_{m}=\hat{\boldsymbol{\psi}}_{1}\left(\mathbf{P}_{m}^{T} \hat{\boldsymbol{\psi}}_{1}\right)^{+} \mathbf{y}_{m}
$$

which is then removed from the measurements,

$$
\mathbf{y}_{m}^{\prime}=\mathbf{y}_{m}-\mathbf{P}_{m}^{T} \hat{\mathbf{x}}_{m}
$$

The deflated measurements $\left\{\mathbf{y}_{m}^{\prime}\right\}_{m=1}^{M}$ are then fed back into (2), and the process is repeated $L-1$ times to approximate $L$ principal components $\left\{\hat{\boldsymbol{\psi}}_{l}\right\}_{l=1}^{L}$ and to produce final reconstruction

$$
\hat{\mathbf{x}}_{m}=\hat{\mathbf{\Psi}}\left(\mathbf{P}_{m}^{T} \hat{\mathbf{\Psi}}\right)^{+} \mathbf{y}_{m},
$$

where $\hat{\boldsymbol{\Psi}}=\left[\begin{array}{lll}\hat{\boldsymbol{\psi}}_{1} & \cdots & \hat{\boldsymbol{\psi}}_{L}\end{array}\right]$

\section{DECENTRALIZED RECONSTRUCTION}

The centralized reconstruction presented above focuses computational burden on a single central node which performs (2)-(5) for the entire dataset. Additionally, the network is burdened by substantial communication, as all nodes must transmit their $\mathbf{y}_{m}$, along with their $\mathbf{P}_{m}$, to this fusion center. The resulting computation and memory load on the fusion-center node, as well as the communication load on the network, may not be practical — or even possible — if the number of sensors $M$ is large.

Fortunately, it is possible to devise a decentralized reconstruction in which each node in the network performs computations involving its own local data $\left(\mathbf{y}_{m}\right.$ and $\left.\mathbf{P}_{m}\right)$ as well as a small amount of data from a limited number of neighboring nodes. This reconstruction hinges on a decentralized implementation of the covariance calculation of (2), the one process that requires aggregation of all data across the network. We propose to effectuate such a decentralized version of (2) with an iterative consensus algorithm for averaging (e.g., [14]). 
Specifically, let the approximation to $\hat{\boldsymbol{\Sigma}}$ at node $m$ for iteration $j$ be $\hat{\boldsymbol{\Sigma}}_{m}^{(j)}$. In a synchronous fashion, each node simultaneously updates its estimate as

$$
\hat{\boldsymbol{\Sigma}}_{m}^{(j+1)}=\hat{\boldsymbol{\Sigma}}_{m}^{(j)}+\sum_{m^{\prime} \in \mathcal{N}_{m}} W_{m, m^{\prime}}\left(\hat{\boldsymbol{\Sigma}}_{m^{\prime}}^{(j)}-\hat{\boldsymbol{\Sigma}}_{m}^{(j)}\right)
$$

where $\mathcal{N}_{m}$ is the set of nodes which are neighbors to node $m$, and $W_{m, m^{\prime}}$ is a weight. Although there are a number of ways to implement the weights, here, we use local-degree weights [14],

$$
W_{m, m^{\prime}}=\frac{1}{\max \left\{\left|\mathcal{N}_{m}\right|,\left|\mathcal{N}_{m^{\prime}}\right|\right\}},
$$

which can be determined by each node with only limited communication with its neighbors. ${ }^{1}$ It has been shown [14] that iterative calculation of (6) will converge to the result of (2),

$$
\lim _{j \rightarrow \infty} \hat{\Sigma}_{m}^{(j)}=\hat{\boldsymbol{\Sigma}}
$$

such that all nodes eventually produce the same covariance as would be used by the central node in the centralized reconstruction outlined in Sec. 2. Each node can then separately calculate the first eigenvector $\hat{\psi}_{1}$ and perform the deflation procedure of (3)-(4) to produce its new measurement $\mathbf{y}_{m}^{\prime}$. This process is repeated for all $L$ eigenvectors, using a new application of the decentralized covariance (6) each time. Finally, each node applies (5) separately to produce its reconstructed vector $\hat{\mathbf{x}}_{m}$. We note that, in (6), it suffices to calculate only the upper-triangular part of the covariance matrix, as each node can complete the rest of the matrix using symmetry.

While the nodes of a real sensor network would implement (6) as written, in the experimental results that follow, we instead merely simulate its network-wide effect over $J$ consensus iterations. Specifically, we define $W_{m, m^{\prime}}=0$ for $m^{\prime} \notin \mathcal{N}_{m}$, such that we collect the weights into $M \times M$ matrix $\mathbf{W}$. Then, we define the $1 \times N(N+1) / 2$ vector $\mathbf{z}_{m}^{(j)}$ as the upper-triangular part of matrix $\hat{\boldsymbol{\Sigma}}_{m}^{(j)}$ reordered as a row vector, assembling all $M$ of these vectors as the rows of $M \times N(N+1) / 2$ matrix $\mathbf{Z}^{(j)}$. Then, the network-wide state at iteration $J$ is

$$
\mathbf{Z}^{(J)}=\mathbf{W}^{J} \mathbf{Z}^{(0)}
$$

where we initialize with $\mathbf{z}_{m}^{(0)}=\mathbf{P}_{m} \mathbf{y}_{m} \mathbf{y}_{m}^{T} \mathbf{P}_{m}^{T}$ at each node [14]. However, when $M$ is large, the matrix power in (9) may be impractical to compute, in which case, we approximate it as

$$
\mathbf{W}^{J} \approx \mathbf{V D}^{J} \mathbf{V}^{T}
$$

where $\mathbf{D}$ is a diagonal matrix containing the largest $d$ eigenvalues of $\mathbf{W}$, and the columns of $\mathbf{V}$ are the corresponding eigenvectors. Below, we use $d=10$.

\footnotetext{
${ }^{1}$ Note that (6) and (7) require that each node be aware of its connections with only its immediate neighbors and not the topology of the network as a whole.
}

As a final note, we observe that [15] also proposes a decentralized covariance calculation; however, in contrast to the "outer-product" covariance (size $N \times N$ ) produced by (2), [15] considers the "inner-product" covariance (size $M \times M)$. A decentralized calculation of the latter is somewhat more complicated than (6) and involves a decentralized power method.

\section{EXPERIMENTAL RESULTS}

We now experimentally compare the proposed decentralized reconstruction of Sec. 3 with the usual centralized reconstruction of Sec. 2. Decentralized processing takes place in a sensor network consisting of $M=10,000$ nodes. In this network, each node is connected to at least two neighbor nodes in order to ensure that the network is strongly connected; additional edges are added at random so that the average number of neighbors (i.e., the expected degree) for the nodes is 3 (i.e., $\left.E\left[\left|\mathcal{N}_{m}\right|\right]=3\right)$. The data at each node in the sensor network takes the form of a single vector with dimensionality reduced via a random projection. As example data, we use pixel vectors extracted from a single hyperspectral image; for this, we employ the well-known AVIRIS images "moffett," "jasper," and "cuprite," each having an original spectral vector dimension of $N=224$. This dimensionality is reduced to $K=67$ (i.e., a subrate of $K / N=30 \%$ ) by orthonormal measurement matrix $\mathbf{P}_{m}$ chosen randomly for each node.

Fig. 1(a) shows the quality of reconstruction in the form of a vector-based signal-to-noise ratio (SNR) [16] averaged over all vectors of the network for the "moffett" dataset. In Fig. 1(a), we vary the number of iterations, $J$, devoted to the average-consensus process ((6), simulated as (9)) at the heart of the proposed decentralized reconstruction; $J$ is directly related to both the time it takes for the sensor network to arrive at the reconstruction as well as the total communication bandwidth expended in doing so. We see that a single iteration of average consensus produces a reconstruction within $2.5 \mathrm{~dB}$ of the centralized reconstruction, with the decentralized performance approaching to within $0.15 \mathrm{~dB}$ of the centralized performance after 100 iterations. Similar results are seen for "jasper" and "cuprite" in Figs. 1(b) and (c), respectively. As a point of reference, a centralized CS-based reconstruction using the multi-task Bayesian CS algorithm of [13] produces SNRs of $18.9 \mathrm{~dB}, 17.9 \mathrm{~dB}$, and $14.9 \mathrm{~dB}$ for the "moffett," "jasper," and "cuprite" data, respectively.

Similar convergence to centralized performance is also exhibited in the angle of difference, $\xi_{l}$, between eigenvector $\hat{\psi}_{l}$ produced by the reconstruction and the true eigenvector $\psi_{l}$ of the data. For example, Fig. 2 depicts $\xi_{l}$ for the first three eigenvectors of the "moffett" data. We see that, after 100 iterations, the decentralized eigenvectors are nearly identical to those produced by the centralized algorithm. 


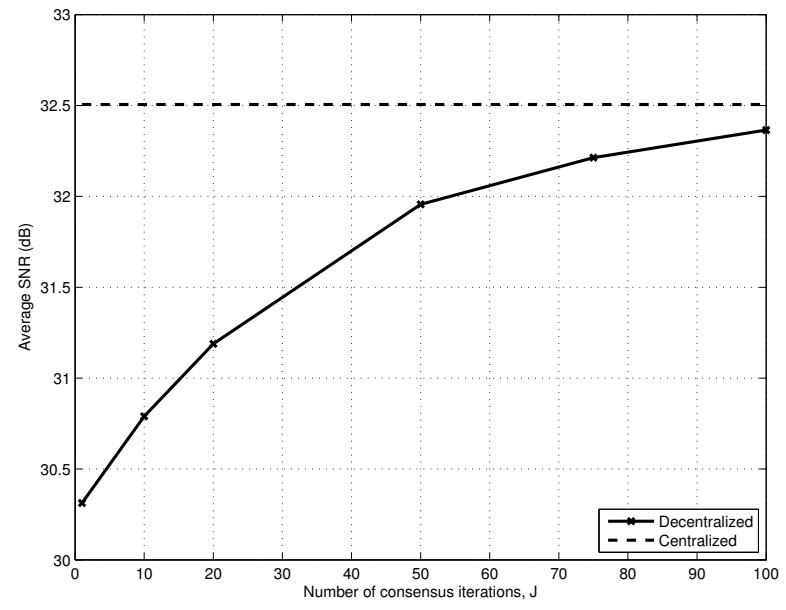

(a) Moffett

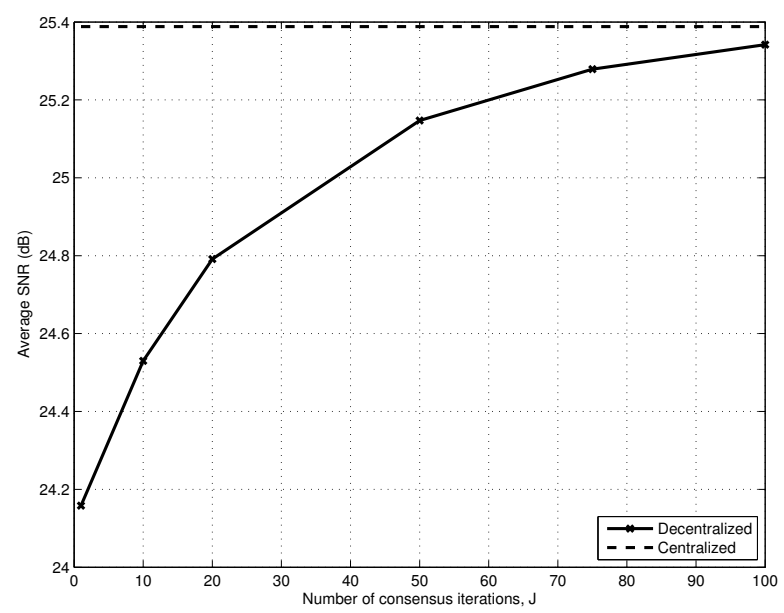

(b) Jasper

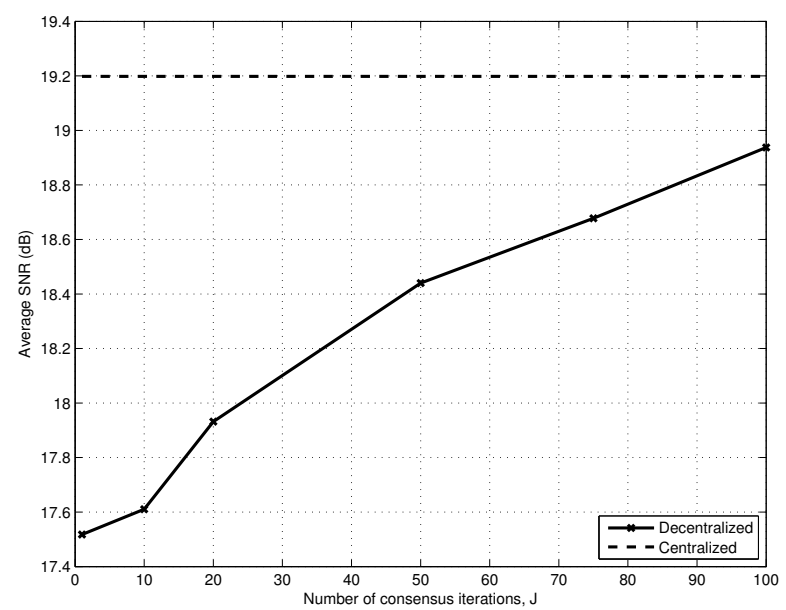

(c) Cuprite

Fig. 1. Average SNR for centralized and decentralized reconstructions as the number of consensus iterations $J$ varies.

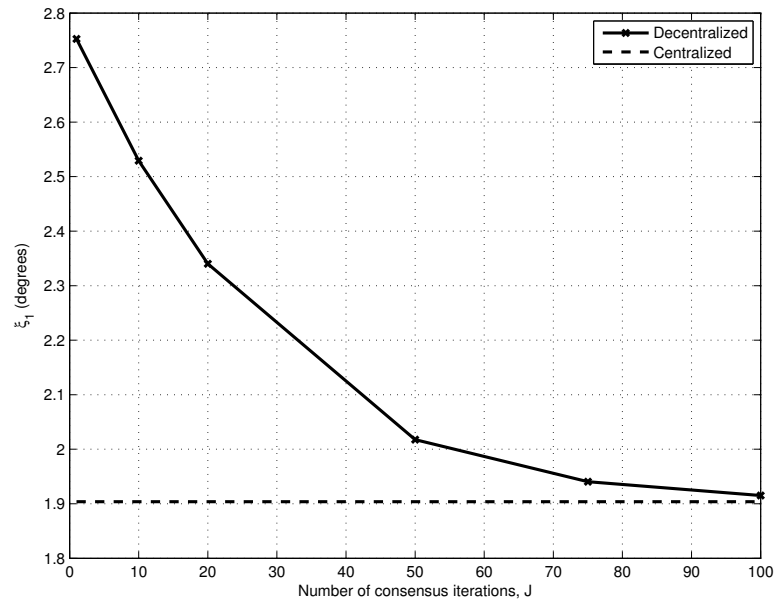

(a) First eigenvector

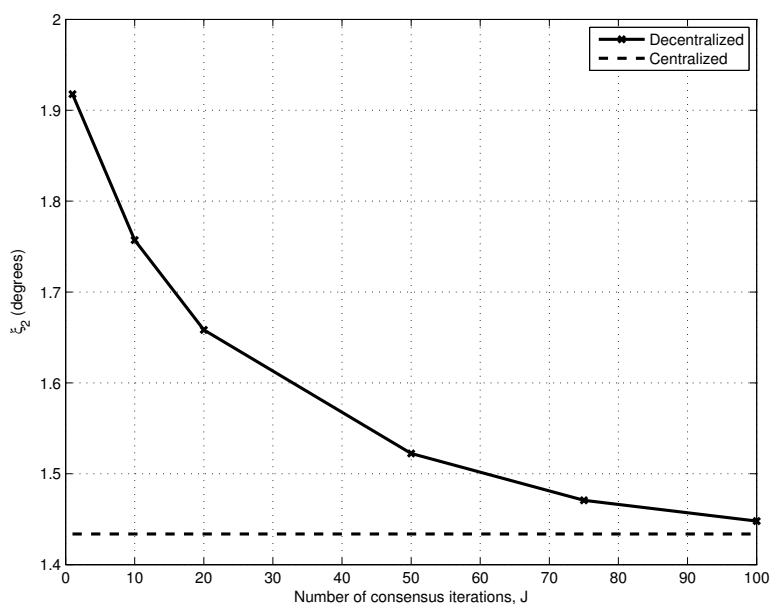

(b) Second eigenvector

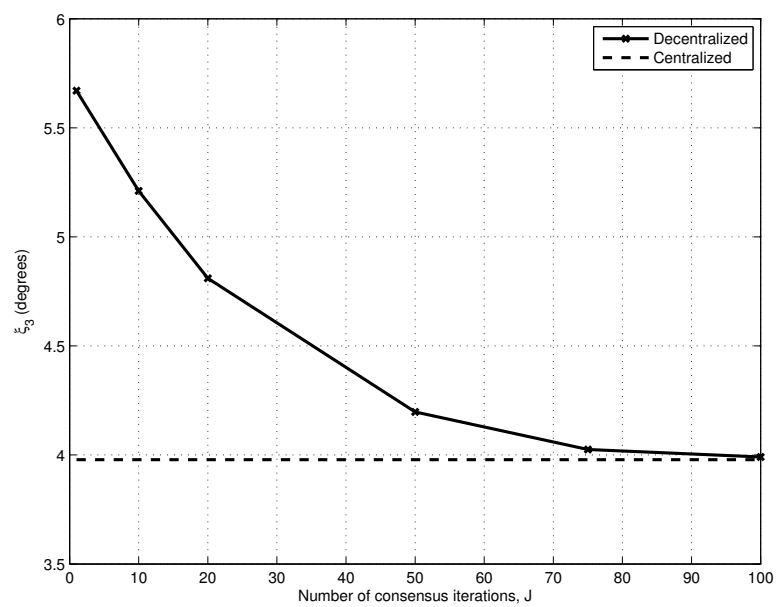

(c) Third eigenvector

Fig. 2. Angle $\xi_{l}$ between reconstructed eigenvector and true eigenvector as the number of consensus iterations $J$ varies. 


\section{CONCLUSION}

In this paper, we proposed a decentralized implementation of the PCA-based reconstruction of [2]. Assuming that individual nodes of a sensor network each acquire a signal and simultaneously reduce its dimensionality by employing a compressive random projection within the sensor device, the proposed algorithm performs a reconstruction whose calculation is distributed throughout the sensor network, with each node performing limited computation with limited communication with its neighboring nodes. At the heart of this decentralized reconstruction is the implementation of a covariance estimation via an iterative consensus algorithm which permits each node of the network to arrive at an approximation of the PCA basis for the entire collection of data distributed across the nodes. Experimental results reveal that, as the number of iterations of consensus increases, the performance of the decentralized reconstruction approaches that of the original centralized algorithm, with 100 iterations typically being sufficient to produce nearly identical results.

\section{REFERENCES}

[1] J. E. Fowler, "Compressive-projection principal component analysis," IEEE Transactions on Image Processing, vol. 18, no. 10, pp. 2230-2242, October 2009.

[2] H. Qi and S. M. Hughes, "Invariance of principal components under low-dimensional random projection of the data," in Proceedings of the International Conference on Image Processing, Orlando, FL, October 2012, pp. 937-940.

[3] M. F. Duarte, S. Savrvotham, D. Baron, M. B. Wakin, and R. G. Baranuik, "Distributed compressed sensing of jointly sparse signals," in Proceedings of the $39^{\text {th }}$ Asilomar Conference on Signals, Systems, and Computers, Pacific Grove, CA, October 2005, pp. 1537-1541.

[4] P. Viñelas-Peris and A. Artés-Rodríguez, "Sensing matrix optimization in distributed compressed sensing," in Proceedings of the IEEE/SP $15^{\text {th }}$ Workshop on Statistical Signal Processing, Cardiff, Wales, August 2009, pp. 638-641.

[5] D. Valsesia, G. Coluccia, and E. Magli, "Joint recovery algorithms using difference of innovations for distributed compressed sensing," in Proceedings of the $47^{\text {th }}$ Asilomar Conference on Signals, Systems, and Computers, Pacific Grove, CA, November 2013, pp. 414-417.

[6] G. Coluccia, A. Roumy, and E. Magli, "Operational rate-distortion performance of single-source and distributed compressed sensing," IEEE Transactions on
Communications, vol. 62, no. 6, pp. 2022-2033, June 2014.

[7] S. Patterson, Y. C. Eldar, and I. Keidar, "Distributed compressed sensing for static and time-varying networks," IEEE Transactions on Signal Processing, vol. 62, no. 19, pp. 4931-4946, October 2014.

[8] _ _ "Distributed sparse signal recovery for sensor networks," in Proceedings of the International Conference on Acoustics, Speech, and Signal Processing, Vancouver, Canada, May 2013, pp. 4494-4498.

[9] J. F. C. Mota, J. M. F. Xavier, P. M. Q. Aguiar, and M. Püschel, "Distributed basis pursuit," IEEE Transactions on Signal Processing, vol. 60, no. 4, pp. 19421956, April 2012.

[10] —, "D-ADMM: A communication-efficient distributed algorithm for separable optimization," IEEE Transactions on Signal Processing, vol. 61, no. 10, pp. 2718-2723, May 2013.

[11] D. Sundman, S. Chatterjee, and M. Skoglund, "A greedy pursuit algorithm for distributed compressed sensing," in Proceedings of the International Conference on Acoustics, Speech, and Signal Processing, Kyoto, Japan, March 2012, pp. 2729-2732.

[12] — , "Parallel pursuit for distributed compressed sensing," in Proceedings of the IEEE Global Conference on Signal and Information Processing, Austin, TX, December 2013, pp. 783-786.

[13] S. Ji, D. Dunson, and L. Carin, "Multitask compressive sensing," IEEE Transactions on Signal Processing, vol. 57, no. 1, pp. 92-106, January 2009.

[14] L. Xiao and S. Boyd, "Fast linear iterations for distributed averaging," in Proceedings of the $42^{\text {nd }}$ IEEE Conference on Decision and Control, Maui, HI, December 2003, pp. 4997-5002.

[15] A. Scaglione, R. Pagliari, and H. Krim, "The decentralized estimation of the sample covariance," in Proceedings of the $42^{\text {th }}$ Asilomar Conference on Signals, Systems, and Computers, Pacific Grove, CA, October 2008, pp. 1722-1726.

[16] J. E. Fowler and Q. Du, "Reconstructions from compressive random projections of hyperspectral imagery," in Optical Remote Sensing: Advances in Signal Processing and Exploitation Techniques, S. Prasad, L. M. Bruce, and J. Chanussot, Eds. Springer, 2011, ch. 3, pp. 31-48. 